\title{
The Role of VEGF and VEGF Receptors in Carcinogenesis
}

\author{
Kevin J. French* and Kendall S. Frazier \\ GlaxoSmithKline, Department of Safety Assessment, UE0376, 709 Swedeland Rd. PO Box 1539, King of Prussia, USA
}

\begin{abstract}
The vascular endothelial growth factor (VEGF) family and its associated cognate receptors are critical components of angiogenesis, the process of new blood vessel formation. Under normal conditions, angiogenesis supports organism development and tissue homeostasis in a tightly controlled fashion. However, in the tumor microenvironment, several conditions, such as hypoxia and unchecked growth factor expression can lead to tumor angiogenesis, enabling endothelial proliferation and vessel assembly, which support cancer growth. Not only does tumor angiogenesis enable tumor proliferation, but its imbalance of regulators cause abnormal vascular structure, which can increase chemoresistance during therapy. Lymphangiogenesis, or the metastases-driven angiogenesis in the lymphatic system, has recently been identified as an essential process for tumor spread to lymph nodes and is driven through unique members of the VEGF signaling pathway family. Inhibition of tumor angiogenesis as an anti-cancer therapeutic strategy has resulted in the development of several recently approved drugs, unfortunately accompanied by angiogenic-related safety issues and off-target associated liabilities unique to individual drug profile. This review summarizes the role of the VEGF and VEGFR families in tumor biology and the therapies available that target these angiogenic pathways.
\end{abstract}

Keywords: Vascular endothelial growth factor; VEGF; Carcinogenesis; Angiogenesis; Lymphangiogenesis

\section{Introduction}

Angiogenesis is the process of new blood vessel formation, and in relationship to cancer, is necessary for neoplastic growth and metastasis, as well as overall tumor cell survival and neoplastic progression. Among the myriad of factors responsible for tumor angiogenesis, the most critical to these processes are members of the vascular endothelial growth factor (VEGF) family, which includes VEGF-A, -B, -C, -D, $-\mathrm{E},-\mathrm{F}$, placental growth factor-1 and -2 (PIGF-1, PIGF-2) and their associated cognate receptors VEGFR-1, VEGFR-2, and VEGFR-3 [1]. Of these ligands, VEGF-A is the most important and relevant in oncology and cancer biology, interacting with both VEGFR-1 and VEGFR-2. A variety of pro-angiogenic mechanistic effects have been ascribed to VEGF-A, including increased vascular permeability, endothelial cell survival, tube formation and sprouting of new vessels, endothelial migration via altered adhesion molecule expression and dissolution of the extracellular milieu via upregulation of serine proteases and matrix metalloproteinases (MMPs). Angiogenesis involves the interplay between pro- and anti-angiogenic growth factors, and mutations of oncogenes and tumor suppressor genes have been shown to alter this homeostasis in favor of angiogenesis in solid tumors. When pro-angiogenic factors such as VEGF predominate, tumors may acquire an angiogenic phenotype leading to endothelial proliferation, migration and differentiation into newly formed vessels. As early as the 1970s, pioneering work by the late Dr. Judah Folkman suggested that targeting the tumor microenvironment using strategies to block tumor angiogenesis might deprive their blood supply and essential nutrients and therefore result in arrest of tumor growth $[2,3]$ The efforts in the ensuing decades have proven this hypothesis to be correct, but the effects of the VEGF pathway on tumor growth have turned out to be much more complex than simply involving depriving cancer cells of their oxygen supply. Recent research has suggested that VEGF signaling has integral roles in vascular integrity, normalization of vascular architecture, hypoxic responses, tumor immunoreactivity and stem cell recruitment. The importance of VEGF in tumor biology has been confirmed by studies on overexpression of VEGF in several tumor types and its correlation with poor prognosis [4]. VEGF-A levels (in particular the $\mathrm{VEGF}_{206}$ isoform) have been demostrated to be increased in mammary, lung, brain, pancreatic, ovarian, kidney, and bladder carcinomas. Other splice variants have also been identified in hematologic malignancies [5]. VEGF criticality to carcinogenesis has been validated further by the approval of several new antiangiogenic therapeutics, many of which demonstrated efficacy in a variety of cancer types.

\section{Background Biology}

Biologic effects of VEGF-A occur via its interaction with the VEGFR-1 and VEGFR-2 receptors, which are localized to the vascular endothelium. VEGF-B, PIGF-1 and PIGF-2 bind to VEGFR-1, whereas VEGFR-3, which is localized to lymphatic endothelium, binds VEGF-C and VEGF-D . Due to this expression pattern, VEGFR-3 is primarily involved in lymphangiogenesis rather than tumor neovascularization [6]. VEGF-E is an exogenous subtype which is a ligand for VEGFR-2, and VEGF-F is a snake venom derived subtype that also binds VEGFR-2. Since VEGF-A is the most important factor in tumor biology, this review will primarily focus on this isoform. The gene for VEGF-A is located at chromosome 6p21.3, and is encoded by 8 exons separated by 7 introns. VEGF-A is a dimeric glycoprotein which is covalently linked by 2 disulfide bridges, and has many isoforms related to alternative exon splicing of exons 6 and 7 [1]. There are 8 conserved cysteine residues with two forming cross-links and the other 6 forming 3 loop structures [7]. The various isoforms have slightly different biologic

*Corresponding author: Dr. Kevin French, GlaxoSmithKline, Department of Safety Assessment, UE0376, 709 Swedeland Rd. PO Box 1539, King of Prussia, USA, Tel: 610-270-4973; Fax: 610-270-5150; E-mail: Kevin.2.french@gsk.com

Received June 30, 2011; Accepted July 16, 2011; Published July 18, 2011

Citation: French KJ, Frazier KS (2011) The Role of VEGF and VEGF Receptors in Carcinogenesis. J Carcinogene Mutagene S2:002. doi:10.4172/2157-2518.S2-002

Copyright: ( 2011 French KJ, et al. This is an open-access article distributed under the terms of the Creative Commons Attribution License, which permits unrestricted use, distribution, and reproduction in any medium, provided the original author and source are credited. 
properties, which is primarily due to varied bioavailability. Sequence variation in two heparin-binding domains determine whether each isoform is secreted or remainsbound tightly to the extracellular matrix [8]. While both VEGFR-1 and VEGFR-2 can stimulate angiogenesis, and VEGFR-1 has a 10-fold higher binding affinity for VEGF-A than VEGFR-2, it is the interaction with VEGFR-2 that is considered most important for angiogenic functions. VEGF expression is regulated by several different mechanisms and specific factors. It upregulates urokinase, tissue plasminogen activator (tPA) and plasminogen activator inhibitor-1 (PAI-1) and is itself upregulated by the hypoxia inducible factor HIF1a/von Hippel-lindau tumor suppressor gene (VHL) pathway [9]. Low oxygen tension in tumors has been associated with increased metastatic potential and poor survivability of many cancers [10]. The degree of increased expression of HIF1a is also well correlated with increased invasive potential in a variety of tumor types, and is intimately linked to increased VEGF expression [9]. VEGF-A can induce the expression and activity of both nitric oxide (NO) and prostacyclin within tumors, resulting in local vasodilation and increased local blood supply. Inhibition of VEGF signaling has been shown to decrease $\mathrm{NO}$ and result in vasoconstriction and tumor shrinkage. Besides hypoxia, many other growth factors upregulate VEGF mRNA at the transcription level including platelet-derived growth factor (PDGF), epidermal growth factor (EGF), fibroblast growth factor (FGF), keratinocyte growth factor and insulin-like growth factor-1 (IGF-1) [8,11]. After kinase activation of the VEGFR-2 receptor, several signal transduction pathways are activated, including PLC- $\lambda 1$, VEGFR-associated protein (VRAP), Ras GAP, FAK, Sck, Src, Grb2,PI3K, Akt, PKC, Raf, MEK/ERK, MAPK, Nck, Crk,82 Shc, and STAT [5].

VEGF-A stimulates tumor angiogenesis through several mechanisms and at several stages relating to endothelial proliferation and vessel assembly. This includes effects on endothelial mitogenesis, enhanced endothelial proliferation and survival; increased endothelial migration and invasion; increased vascular permeability, and enhanced chemotaxis [12,13]. VEGF-A has been shown to be mitogenic in endothelial cell culture. Stimulation of the VEGF pathway can also induce proliferation in leukemic cells and other tumor types [14]. The effects on endothelial proliferation are concentration dependent with high levels resulting in endothelial growth and division while low levels promote migration during branching morphogenesis [15]. VEGF-A or VEGFR-1 knockout mice demonstrate decreased blood islands in the yolk sac, also suggesting VEGF signaling is required for endothelial proliferation [16]. The binding of VEGF-A to VEGFR-2 causes receptor dimerization, kinase activation and autophosphorylation of specific tyrosine residues within the dimeric complex. DNA synthesis is stimulated through direct phosphorylation of MAPK and stimulation of the ERK1/2 pathway as well as through PKC and PI3K [17]. VEGF-A is a survival factor for endothelial cells and activates Bcl-2, survivin and the inhibitors of apoptosis proteins (IAPs). Inhibition of VEGF can induce endothelial apoptosis through modulation of the PI3K/ $\mathrm{AKT}$ and $\mathrm{Bcl}-2$ pathways [18]. VEGF has been demonstrated to be an essential factor for endothelium in tumor xenografts as well as in vitro and VEGF inhibition can result in increased apoptosis and potential tumor regression through similar mechanisms [19].

VEGF signaling is important in both endothelial and tumor cell chemotaxis [14]. These activities are dependent on Neuropilin-1 (NRP1), a nonkinase cell surface co-receptor involved in cell motility, which forms complexes with and potentiates the binding of VEGF-A to VEGFR-2 [20]. Inhibition of complex formation is associated with reduced VEGFR2 phosphorylation, intracellular signaling, mitogenesis, cell migration and angiogenesis [20-22]. NRP1 also regulates endothelial cell function and cancer proliferation stimulated by other growth factors [23]. Normally, vessels are assembled from endothelial precursors, with the expansion of this primitive vascular network by sprouting, bridging, branching and intussusception (insertion of interstitial tissue columns into the lumen of preexisting vessels) of the newly formed vessels. Angiogenesis is a complex process involving several critical steps including secretion of factors by endothelium or tumor cells, the subsequent migration proliferation and differentiation of endothelium and the assembly of primary vessels. During tumor angiogenesis, recruitment of endothelial cells into newly formed microvessel buds is an important initial step. These endothelial cells can arise via proliferation of endothelium within the tumor or be co-opted from normal vessels in the adjacent parenchyma [24]. In addition, circulating endothelial progenitor cells (cEPCs), which are normally involved in vascular repair, can participate in the development of tumor vasculature as well, and their localization to the angiogenic site is also under the influence of VEGF-mediated chemotaxis [25]. Nitric oxide synthase (NOS) expression and activity appears to be one of the mediators of this process. Phosphorylated VEGFR-2 binds phospholipase Cy1 (PLC- $\gamma 1$ ) and Src homology 2 domain containing adaptor protein $B(\mathrm{Shb})$. Activation of PLG- $\gamma 1$ and Shb regulates VEGF-A-dependent cell proliferation/mitogenesis and cell migration, respectively. Phosphorylated VEGFR-2 also binds and mediates tyrosine phosphorylation of the T-cell-specific adaptor protein (TSAd), which is expressed in endothelial cells and is believed to be critical for VEGF-A-induced chemotaxis and actin reorganization [26]. VEGF signaling has been demonstrated to be chemotactic for multiple tumor types, including glioma and hematologic malignancies. VEGF-A has shown to be particularly important in the chemotactic response associated with cEPCs and hematopoietic precursors. VEGF is required for organ homing and perivascular positioning of circulating mononuclear myeloid cells to angiogenic vessels, and this response as well as many other homing activities of circulating progenitor cell populations appear to be mediated by interactions with stromalderived factor-1 (SDF1), a chemokine induced by VEGF in activated perivascular myofibroblasts [27]. These and other recent clinical data support the concept that tumor infiltration by bone marrow-derived myeloid cells confers resistance to current anti-angiogenic drugs targeting primarily VEGF. Chemoattractants recruit hematopoietic cells from bone marrow that support tumor neovascularization in the primary cancer site, and these mobilized cells are thought to participate in pre-metastatic niche formation for circulating tumor cells. Integration of bone marrow cells into the tumor vasculature as a late event in carcinogenesis correlates with VEGF release by the tumor and mobilization of circulating endothelial progenitor cells. For example, hematopoietic progenitor cell populations have shown to be attracted to glioma brain tumor cells and this response appears to be mediated in part through VEGF/VEGF-2 induced CD62E expression [28]. Other "normal" cells may be recruited under conditions of tumor angiogenesis and chemotaxis to sites of metastasis by soluble products such as VEGF-A released by cancer cells or tumor endothelium, and these cells have been shown to create or pattern a microenvironment or niche favorable to tumor cell proliferation and survival and potentially to activate poorly angiogenic tumor cells seeded to these sites of metastasis [29]. Thus, cross-communication between tumor and host cells mediated by VEGF and other chemoattractants may alter the 
proinflammatory milieu of both primary and metastatic lesions in favor of cancer progression [30].

After cEPC recruitment, another important aspect of angiogenesis involves modulation of the extracellular matrix to enhance the framework for building new vessels. The vasculature becomes increasingly permeabilized under the influence of VEGF-A, resulting in tissue edema. Detachment of pericytes is associated with this permeabilization effect and has also been demonstrated to be under VEGF influence [31]. This is followed by the depositin of fibrin gels in the tissue bed, which allows endothelial growth and vessel prolongation. Proteolytic degradation of the extracellular milieu and the noncompliant basal lamina is an important prerequisite, and this is dependent on matrix metalloproteinases (MMPs) and serine proteases secreted by endothelial cells in the sprouting tips of growing vessels, under the influence of VEGF-A. Several metalloproteases have been shown to be upregulated by VEGF-A isoforms in angiogenic models. Interestingly, tissue inhibitor of metalloproteinases-2 (TIMP-2) inhibits the mitogenic response of endothelial cells to VEGF-A in vitro and in vivo, independent of metalloproteinase inhibition [32]. VEGF has also been shown to increase the activity of cathepsins in sprouting venules and down regulate the activity of cysteine protease inhibitors. This disrupts the normal cathepsin-inhibitor balance, leading to degradation of their basement membranes [33]. The upregulation of proteases by VEGF and excessive MMP activity can also facilitate neoplastic progression directly by increasing the local invasiveness of cancers into the surrounding parenchyma as well as by increasing metastases [32]. The balance between extracellular matrix degrading enzymes and their endogenous inhibitors determines the extent of tissue remodeling, and the increase in protease activity in relationship to local protease inhibitors results in a microenvironment favorable to tumor progression.

It is well established that the formation of the mature vascular network within tumors is incomplete and therefore the structure of neoplastic vasculature is abnormal. The network of tumor vessels is unorganized without a clear separation of arterial and venous systems, and abnormalities in the vessel wall, and basement membrane have been consistently identified [34]. The imbalance of angiogenic regulators and predominance of VEGF-A account for this abnormal structure, which in turn results in chaotic blood flow and vessel permeability [35]. These alterations result in increased intestitial fluid pressures within the tumor and hence the presence of hypoxic regions that can further upregulate the HIF1a-VEGF-A axis [36]. Altered intra-tumoral blood flow has been shown to increase the resistance of tumors to chemotherapy via several mechanisms, including lower drug delivery and selection of more malignant phenotype associated with altered cell cycles and enhanced mutagenesis. Many of the clinical benefits of inhibition of VEGF-A using antibody or tyrosine kinase inhibitors (TKIs) in patients is now thought to be through transient normalization of tumor microvasculature into a pattern that mimics that of more normal physiology occurring in angiogenesis of wound healing. This is hypothesized to improve drug and oxygen delivery to tumor cells, making them more chemosensitive and radiosensitive. Unfortunately, this window of normalization is transient and relatively short, at least in mice [37]. There also appears to be a slow shift in the angiogenic dependence of tumors over time clinically, with tumors switching from VEGF dependent pathways to alternative mechanisms $[24,29]$. This may result in eventual decreased clinical responses in many patients. Together, these observations of transient normalization and alterations of VEGF dependence in the tumor microvasculature have been suggested to help explain the relatively good responses in progession free survival but somewhat less robust long term survival rates noted with VEGF-targeted single agent cancer therapies currently on the market $[38,39]$. However, the decreased dependence on VEGFmediated angiogenesis should not be confused with true tumor resistance, as these chemotherapeutic agents are targeting the stromal microvasculature rather than the tumor itself. This concept is supported by anecdotal evidence from several clinical trials with various agents that indicate there are often flare ups in tumor growth after withdrawal of the anti-VEGF agents, suggesting some inhibitory effect on tumor growth even after progression.

In addition to effects on endothelium and angiogenesis, VEGF signaling has independent functions that affect tumor cell survival and invasion, as well as effects on immune regulation and homing of bone marrow progenitor populations. VEGF is a pivotal factor in hematopoiesis, which specifically affects the differentiation of multiple hematopoietic lineages and it triggers growth, survival, and migration of leukemia and multiple myeloma cells, all relevant to the potential malignant progression of hematologic malignancies [5]. Upregulation of Bcl-2 and survivin are also likely responsible for the increased survival/decreased apoptosis noted with VEGF-A in various tumor cell lines and xenografts. Some of these apoptosis pathways are specific for particular tumors or conditions. For instance, an autocrine VEGF-A/ VEGFR-2 loop has been demonstrated in epithelial ovarian carcinoma cells which appears to play a role in cancer survival, specifically protecting the cells from apoptosis under conditions of anchorage-free growth conditions [40]. VEGF blocking agents might thus potentially reduce peritoneal dissemination by both decreasing vascular permeability and inhibiting aberrant cell survival of detached cancer cells.

In relationship to its immune functions, there are a variety of VEGFmediated effects due to its interrelationship with cytokine expression. High levels of VEGF-A expression have been demonstrated in the proliferative phase of wound healing indicating a functional role in the inflammatory process, and potentially related to neovascularization associated with granulation tissue formation. Dysregulated angiogenesis has been observed in several inflammatory diseases such as psoriasis or rheumatoid arthritis and VEGF is thought to play a role in the pathogenesis of some chronic inflammatory conditions [41]. For instance, transgenic over-expression of VEGF in keratinocytes in mice results in chronic cutaneous inflammation [42]. Given the importance of inflammation in tumor immunosurveillance as well as the paradoxical effects on tumor promotion, secondary inflammatory effects of VEGF signaling may have opposing effects on carcinogenesis related to these pathways. Recent epidemiological and clinical data have supported the concept that chronic inflammation may promote tumor development, growth and progression [43]. While the histological presence of inflammation may be a positive sign of immune rejection in a few types of tumors, the presence of chronic inflammation in breast and prostate tissues is thought to play a role in cancer development, and multiple inflammatory genes including VEGF-A and VEGFR-1 have been associated with prostate cancer risk [44]. Among other proinflammatory gene products involved in such interactions are tumor necrosis factor alpha (TNF- $\alpha$ ) and interleukin 6 (IL-6). In association with VEGF-A, these cytokine interactions may stimulate downstream signal transduction pathways related to tumor progression. Clinically, 
several reports have detected abnormally high levels of these circulating cytokines and growth factors in cancer patients [43]. Macrophages are an abundant inflammatory cell type in the tumor microenvironment, and likely to contribute to tumor growth and metastasis. Macrophage recruitment into tumors is mediated by multiple cytokines, including VEGF-A, which is thought to function primarily through VEGFR-1 in this cell type [45]. Macrophage infiltration is negatively affected by VEGF inhibition and selective inhibition of VEGFR2 has shown to reduce macrophage infiltration into tumors [45]. VEGF may also interact with or be secreted by other inflammatory cell types at the site of tumors such as mast cells [46].

\section{Lymphangiogenesis}

While VEGF-A and its receptor VEGFR-2 have received much of the attention in the field of carcinogenesis, the role of VEGF-C and VEGFR-3 in lymphangiogenesis also has important implications in cancer progression. Lymphangiogenesis is critically involved in cancer recurrence and lymphatic endothelial cell proliferation and lymphatic remodeling regulated by lymphangiogenic factors actively promote tumor metastasis and the inflammation process [47]. Malignant tumors can not only utilize the lymphatic system for metastasis, but may actively induce the formation of new lymphatic vessels to facilitate this process [48]. VEGF-A with VEGFR-2 and VEGF-C/VEGF-D with their receptor VEGFR-3 have all been implicated as mediators in tumor-induced lymphangiogenesis, and their effects can be initiated both within the tumor and within draining lymph nodes [49]. Recent studies indicate that blockade of the lymphangiogenic growth factor signaling pathways with anti-VEGF-C or anti-VEGFR-3 modalities inhibit tumor spread to lymph nodes in bladder and other cancers [50,51]. Upregulation of VEGF lymphangiogenic factors has also been demonstrated in some cancer patients. The mechanism of effects on cancer promotion involving lymphangiogenesis probably parallels that of the VEGF-mediated effects in tumor angiogenesis, and may be related to not only vascular support, but other similar effects like degradative effects on the extracellular matrix, lymph vessel permeability and tumor or stem cell chemotaxis. Pluripotent mesenchymal stem cells migrate along a VEGF-C gradient, and have been demonstrated to develop a lymphendothelial phenotype under VEGF-C influence [47].

\section{Inhibitors of VEGF and VEGF-R and their role as anticancer agents}

The initial discoveries described above linking VEGF, VEGF-R and tumor angiogenesis fostered significant advances in targeted therapy against multiple tumor types. An early example of this is the discovery of the dependence of renal cell carcinoma (RCC) on VEGF. In the majority of RCC cases, the von Hippel-Lindau (VHL) gene is inactivated $[52,53]$. VHL is a tumor suppressor that negatively regulates hypoxia-inducble factor-alpha ( $\mathrm{HIF} \alpha)$, a regulator of hypoxic response. Unchecked levels of HIF- $\alpha$ results in trascriptional activation of hypoxia-inducible genes, which include VEGF and PDGF and contribute to a vascularized state, making VHL-negative RCC tumors ideal candidates for antiangiogenic therapy. In metastatic colorectal cancer (mCRC), overexpression of VEGF has been associated with tumor progression and poor prognosis [54,55], and circulating VEGF levels has correlated with tumor stage [56]. Similar correlates between VEGF tumor expression and circulating VEGF levels have been identified in other "vascular" cancers, most commonly in nonsmall cell lung cancer [57], breast cancer [58], ovarian cancer [59], and gliomas [60], providing strong rationale for VEGF pathway inhibition.
Several antiangiogenic strategies have proven successful, the earliest manifested by interfering with VEGF binding to its cognate receptor. The first antiangiogenic therapy to demonstrate clinical efficacy and regulatory approval via this approach was bevacizumab, a humanized monoclonal antibody directed against VEGF. The United States Food and Drug Administration approved bevacizumab for mCRC in 2004, with subsequent approvals in other cancers, including nonsmall cell lung cancer (NSCLC), HER-2-negative breast cancer, glioblastoma (GSB) and RCC. Preclinically, bevacizumab monotherapy was shown to inhibit VEGF-induced neovascularization events, including proliferation of endothelial cells and tumor angiogenesis, as well as decreasing the growth of various human tumor xenografts in mice [61]. However, efficacy was even more pronounced when adminstered in combination with chemotherapeutic agents, which is believed to be due to distinct mechanisms of action and the ability of VEGF inhibiton to enable vascular access to tumors. The preclinical findings correlated well with later clinical results, enabling the multiple approvals described above. However, the recent FDA committee decision to withdraw bevacisumab for breast cancer [62] highlights both the tumor type dependence on antiangiogenic therapy and need to continually monitor emerging clinical results.

Inhibiting the tyrosine kinase activity of VEGF-R, which is essential for effective angiogenic cell signaling, has also been effective, with regulatory approvals for sorafenib against RCC in 2005 and later against hepatocellular carcinoma (HCC), sunitinib against RCC and gastrointestinal stromal tumors (GIST) in 2006 and pazopanib against RCC in 2009. Sorafenib is a Raf kinase inhibitor which inhibits VEGFR-1,-2, -3, PDGFR- $\beta$, Fms-like tyrosine kinase-3 (Flt-3), and c-kit. Sunitinib inhibits VEGFR-1, -2 and -3 , PDGFR- $\alpha$ and $\beta$, c-kit, Flt-3, colony stimulating factor Type 1 , and the glial cell-line derived neurotrophic factor receptor (RET). Pazopanib inhibits VEGFR-1, -2 and -3 , PDGFR- $\alpha$ and $-\beta$ and $c$-kit. These potencies against multiple kinases allow the above compounds to not only disrupt tumor vasculature, but also inhibit tumor survival signaling pathways. For instance, activating mutations of c-kit or PDGFR- $\alpha$ occur in the majority of cases of GIST and are more responsive in vitro as compared to tumors with less sensitive mutations [63]. In addition, sunitinib was shown as a single agent to inhibit growth of cancer cell lines expressing kit, flt-3 and ret with activating mutations [64]. Similarly, sorafenib inhibited growth of cancer cell lines with oncogenic k-ras, b-raf and ret mutations [65], and pazopanib showed modest activity in a wide variety of human cell lines obtained from AML, myeloma, colon carcinoma, kidney leiomyoblastoma, thyroid carcinoma, rhabdomyosarcoma, CML, lymphoma cutaneous Tcell, and stomach carcinoma [66]. It is most likely that the small molecule antiangiogenic drugs' demonstrated efficacy as monotherapy, and in varied and unique tumor types in vitro and in clinical trials occurs through the multiple mechanisms described above.

While inhibiting VEGF or VEGFR has led to great advances in cancer therapy, safety concerns have also been identified. Adverse events commonly noted in patients clinically with virtually all of these antiangiogenic therapies targeting the VEGF pathway include diarrhea, fatigue and hypertension. Hypertension has been noted with incidences of 5-18\% of patients treated with bevacizumab [67], and at significant incidence in RCC patients given either sorafenib [68], sunitinib or pazopanib [69]. Hypertension has been shown to be a mechanistic, on-target effect of VEGF signaling inhibition and necessitates blood pressure monitoring of patients on therapy. When added exogenously, 
VEGF administration elicits a vasodilatory effect via NO-dependent pathway [70,71], which can be pharmacologically inhibited with antiangiogenic therapy [72]. Other class-wide overlapping adverse events between biologic and small molecule therapies targeting VEGF and VEGFR include gastrointestinal toxicity, proteinuria, and coagulation disorders $[73,74]$.

Exerting both antiangiogenic activity and critical tumor cell kinase activity has the benefit of increased efficacy, but it also leads to additional safety concerns. There are several less common side effects with the antiangiogenic TKIs that vary in incidence among agents and may be related to inhibition of other kinases or related to synergism between anti-VEGF activity and other off target toxicity, including hypothyroidism, hepatic toxicity, hand-foot syndrome or hematologic effects $[67,68]$. An important potential adverse clinical safety concern with some of these multitargeted agents thought to be unrelated to VEGF inhibition that has emerged is cardiotoxicity. Nonoverlapping inhibition of kinases at potencies comparable to clinical exposures have been identifed for sorafenib and sunitinib [75-77]. While both compounds significantly inhibit the VEGF pathway, cardiotoxicity appears to be induced or exacerbated by concurrent inhibition of several other kinase targets. For example, sorafenib is a potent inhibitor of raf-1, which demonstrates antiapoptotic activity in cardiomyocytes through inactivation of Apoptosis signal-regulating kinase 1 (ASK-1) [78]. Through an inhibitor binding assay, sunitinib was shown to inhibit the cardiac prosurvivial proteins ribosomal S6 kinase (RSK) family and AMP-activated protein kinase (AMPK) [79]. Recently, we have identified kinase inhibitor dependent (and potentially antiangiogenesis independent) myocardial effects using a strain rate echocardiography rat model [80]. Such cardiomyocyte alterations may be amplified in patients when combined with the VEGF-mediated hypertensive effects of this class of drugs. Further elucidations into kinase inhibitors' effect on the cardiovascular system will no doubt follow the emerging research into cardiac survival signaling pathways under both normal and stressed conditions.

\section{Conclusions}

VEGF signaling and the outcome of this signaling pathway, angiogenesis, are essential for normal development. These processes are equally essential for progression of many tumor types and therefore represent an exciting target for novel cancer therapeutics. Understanding the role of VEGF mediated tumor-stromal interactions has also led to a greater understanding of the local tumor environment's role in driving metastasis. The successes of targeted therapies for vascular tumor types that depend upon VEGF-A and VEGFR-2 for angiogenesis may provide the momentum for continued exploration into the role of other VEGF family members in lymphangiogenesis and tumor progression. The multitargeted approach to chemotherapy with small molecule antiangiogenic compounds affecting multiple kinase pathways has provided key insights into the specific roles of VEGF in these processes by highlighting synergies with other kinases and magnifying differences among antiangiogenic drugs related to both desired and undesired effects. As additional therapies targeting this pathway are developed, our understanding of the complex role of VEGF in carcinogenesis will continue to broaden.

\section{References}

1. Ferrara N (2004) Vascular endothelial growth factor: basic science and clinical progress. Endocr Rev 25: 581-611.
2. Folkman J (1971) Tumor angiogenesis: therapeutic implications. N Engl J Med 285: $1182-1186$.

3. Folkman $\mathrm{J}(1990)$ What is the evidence that tumors are angiogenesis dependent? J Natl Cancer Inst 82: 4-6.

4. Ferrara N (2005) VEGF as a therapeutic target in cancer. Oncology 3: 11-16.

5. Podar K, Anderson KC (2005) The pathophysiologic role of VEGF in hematologic malignancies: therapeutic implications. Blood 105: 1383-1395.

6. Kumaran GC, Jayson GC, Clamp AR (2009) Antiangiogenic drugs in ovarian cancer. Br J Cancer 100: 1-7.

7. Wiesmann C, Fuh G, Christinger HW, Eigenbrot C, Wells JA, et al. (1997) Crystal structure at 1.7 A resolution of VEGF in complex with domain 2 of the Flt-1 receptor. Cell 91: 695-704.

8. Ferrara N, Davis-Smyth T (1997) The biology of vascular endothelial growth factor. Endocr Rev 18: 4-25.

9. Bos R, Zhong H, Hanrahan CF, Mommers EC, Semenza GL, et al. (2001) Levels of hypoxia-inducible factor-1 alpha during breast carcinogenesis. J Nat Cancer Inst 93: 309-314.

10. Zhong H, De Marzo AM, Laughner E, Lim M, Hilton DA, et al. (1999) Overexpression of hypoxia-inducible factor 1alpha in common human cancers and their metastases. Cancer Res 59: 5830-5835.

11. Neufeld G, Cohen T, Gengrinovitch S, Poltorak Z (1999) Vascular endothelia growth factor (VEGF) and its receptors. FASEB J 13: 9-22.

12. Ellis LM, Hicklin DJ (2008) VEGF-targeted therapy: mechanisms of anti-tumour activity. Nat Rev Cancer 8: 579-591.

13. Rafii S (2002) Vaccination against tumor neovascularization: Promise and reality. Cancer Cell 2: 429-431.

14. Lu L, Zhang L, Xiao Z, Lu S, Yang R, et al. (2008) Neuropilin-1 in acute myeloid leukemia: expression and role in proliferation and migration of leukemia cells. Leuk Lymphoma 49: 331-338

15. Bohnsack BL, Hirschi KK (2004) Red light, green light: signals that contro endothelial cell proliferation during embryonic vascular development. Cell Cycle 3: 1506-1511.

16. Shalaby F, Rossant J, Yamaguchi TP, Gertsenstein M, Wu XF, et al. (1995) Failure of blood-island formation and vasculogenesis in Flk-1-deficient mice. Nature 376: 62-66

17. Yu Y, Sato JD (1999) MAP kinases, phosphatidylinositol 3-kinase, and p70 S6 kinase mediate the mitogenic response of human endothelial cells to vascular endothelial growth factor. J Cell Physiol 178: 235-246.

18. Gerber HP, McMurtrey A, Kowalski J, Yan M, Keyt BA, et al. (1998) Vascula endothelial growth factor regulates endothelial cell survival through the phosphatidylinositol 3'-kinase/Akt signal transduction pathway. Requirement for Flk-1/KDR activation. J Biol Chem 273: 30336-30343.

19. Laird AD, Christensen JG, Li G, Carver J, Smith K, et al. (2002) SU6668 inhibits Flk-1/KDR and PDGFRbeta in vivo, resulting in rapid apoptosis of tumor vasculature and tumor regression in mice. FASEB J 16: 681-690.

20. Wang L, Zeng H, Wang P, Soker S, Mukhopadhyay D (2003) Neuropilin-1 mediated vascular permeability factor/vascular endothelial growth factordependent endothelial cell migration. J Biol Chem 278: 48848-48860.

21. Oh H, Takagi H, Otani A, Koyama S, Kemmochi S, et al. (2002) Selective induction of neuropilin-1 by vascular endothelial growth factor (VEGF): a mechanism contributing to VEGF-induced angiogenesis. Proc Natl Acad Sci U S A 99: 383-388

22. Pan Q, Chanthery Y, Liang WC, Stawicki S, Mak J, et al. (2007) Blocking neuropilin-1 function has an additive effect with anti-VEGF to inhibit tumor growth. Cancer Cell 11: 53-67.

23. Evans IM, Yamaji M, Britton G, Pellet-Many C, Lockie C, et al. (2011) Neuropilin-1 signaling through p130Cas tyrosine phosphorylation is essential for growth factor-dependent migration of glioma and endothelial cells. Mol Cell Biol 31: 1174-1185. 
24. Holash J, Maisonpierre PC, Compton D, Boland P, Alexander CR, et al. (1999) Vessel cooption, regression, and growth in tumors mediated by angiopoietins and VEGF. Science 284: 1994-1998.

25. Heiss C, Schanz A, Amabile N, Jahn S, Chen Q, et al. (2010) Nitric oxide synthase expression and functional response to nitric oxide are both importan modulators of circulating angiogenic cell response to angiogenic stimuli. Arterioscler Thromb Vasc Biol 30: 2212-2218.

26. Matsumoto $T$, Mugishima $\mathrm{H}$ (2006) Signal transduction via vascular endothelia growth factor (VEGF) receptors and their roles in atherogenesis. J Atheroscler Thromb 13: 130-135.

27. Grunewald M, Avraham I, Dor Y, Bachar-Lustig E, Itin A, et al. (2006) VEGFinduced adult neovascularization: recruitment, retention, and role of accessory cells. Cell 124: 175-189.

28. Tabatabai G, Herrmann C, von Kürthy G, Mittelbronn M, Grau S, et al. (2008) VEGF-dependent induction of CD62E on endothelial cells mediates glioma tropism of adult haematopoietic progenitor cells. Brain 131: 2579-2595.

29. Favaro E, Amadori A, Indraccolo S (2008) Cellular interactions in the vascular niche: implications in the regulation of tumor dormancy. APMIS 116: 648-659.

30. Furuya M, Yonemitsu Y (2008) Cancer neovascularization and proinflammatory microenvironments. Curr Cancer Drug Targets 8: 253-265.

31. Hasumi Y, Kłosowska-Wardega A, Furuhashi M, Ostman A, Heldin CH, et al. (2007) Identification of a subset of pericytes that respond to combination therapy targeting PDGF and VEGF signaling. Int J Cancer 121: 2606-2614.

32. Stetler-Stevenson WG (2008) The tumor microenvironment: regulation by MMP-independent effects of tissue inhibitor of metalloproteinases-2. Cancer Metastasis Rev 27: 57-66.

33. Chang SH, Kanasaki K, Gocheva V, Blum G, Harper J, et al. (2009) VEGF-A induces angiogenesis by perturbing the cathepsin-cysteine protease inhibitor balance in venules, causing basement membrane degradation and mother vessel formation. Cancer Res 69: 4537-4544.

34. Paku S, Paweletz N (1991) First steps of tumor-related angiogenesis. Lab Invest 65: 334-346.

35. Morikawa S, Baluk P, Kaidoh T, Haskell A, Jain RK, et al. (2002) Abnormalities in pericytes on blood vessels and endothelial sprouts in tumors. Am J Pathol 160: $985-1000$

36. Heldin $\mathrm{CH}$, Rubin K, Pietras K, Ostman A (2004) High interstitial fluid pressure - an obstacle in cancer therapy. Nat Rev Cancer 4: 806-813.

37. Jain RK (2001) Normalizing tumor vasculature with anti-angiogenic therapy: a new paradigm for combination therapy. Nat Med 7: 987-989.

38. Escudier B, Eisen T, Stadler WM, Szczylik C, Oudard S, et al. (2009) Sorafenib for treatment of renal cell carcinoma: Final efficacy and safety results of the phase III treatment approaches in renal cancer global evaluation trial. J Clin Oncol 27: 3312-3318

39. Miller K, Wang M, Gralow J, Dickler M, Cobleigh M, et al. (2007) Paclitaxel plus bevacizumab versus paclitaxel alone for metastatic breast cancer. $\mathrm{N}$ Engl J Med 357: 2666-2676

40. Sher I, Adham SA, Petrik J, Coomber BL (2009) Autocrine VEGF-A/KDR loop protects epithelial ovarian carcinoma cells from anoikis. Int J Cancer 124: 553561.

41. Canavese M, Altruda F, Ruzicka T, Schauber J (2010) Vascular endothelia growth factor (VEGF) in the pathogenesis of psoriasis--a possible target for novel therapies? J Dermatol Sci 58: 171-176.

42. Detmar M, Brown LF, Claffey KP, Yeo KT, Kocher O, et al. (1994) Overexpression of vascular permeability factor/vascular endothelial growth factor and its receptors in psoriasis. J Exp Med 180: 1141-1146.

43. Guadagni F, Ferroni P, Palmirotta R, Portarena I, Formica V, et al. (2007) Review. TNF/NEGF cross-talk in chronic inflammation-related cancer initiation and progression: an early target in anticancer therapeutic strategy. In Vivo 21 : 147-161.

44. Sun J, Turner A, Xu J, Grönberg H, Isaacs W (2007) Genetic variability in inflammation pathways and prostate cancer risk. Urol Oncol 25: 250-259.
45. Dineen SP, Lynn KD, Holloway SE, Miller AF, Sullivan JP, et al. (2008) Vascular endothelial growth factor receptor 2 mediates macrophage infiltration into orthotopic pancreatic tumors in mice. Cancer Res 68: 4340-4346.

46. Conti P, Castellani ML, Kempuraj D, Salini V, Vecchiet J, et al. (2007) Role of mast cells in tumor growth. Ann Clin Lab Sci 37: 315-322.

47. Ji RC (2009) Lymph node lymphangiogenesis: a new concept for modulating tumor metastasis and inflammatory process. Histol Histopathol 24: 377-384.

48. Da MX, Wu Z, Tian HW (2008) Tumor lymphangiogenesis and lymphangiogenic growth factors. Arch Med Res 39: 365-372.

49. Li L, Liu B, Li X, Yang S, Xiao J, et al. (2009) Vascular endothelial growth factor $\mathrm{D}$ and intratumoral lymphatics as independent prognostic factors in epithelia ovarian carcinoma. Anat Rec (Hoboken ) 292: 562-569.

50. Dong Z, Wei F, Zhou C, Sumida T, Hamakawa H, et al. (2011) Silencing Id-1 inhibits lymphangiogenesis through down-regulation of VEGF-C in ora squamous cell carcinoma. Oral Oncol 47: 27-32.

51. Yang H, Kim C, Kim MJ, Schwendener RA, Alitalo K, et al. (2011) Soluble vascular endothelial growth factor receptor-3 suppresses lymphangiogenesis and lymphatic metastasis in bladder cancer. Mol Cancer 10: 36.

52. Yang H, Kim C, Kim MJ, Schwendener RA, Alitalo K, et al. (1994) Somatic mutations of the von Hippel-Lindau disease tumour suppressor gene in nonfamilial clear cell renal carcinoma. Hum Mol Genet 3: 2169-2173.

53. Goodman MD, Goodman BK, Lubin MB, Braunstein G, Rotter JI, et al. (1990) Cytogenetic characterization of renal cell carcinoma in von Hippel-Lindau syndrome. Cancer 65: 1150-1154.

54. Lee JC, Chow NH, Wang ST, Huang SM (2000) Prognostic value of vascula endothelial growth factor expression in colorectal cancer patients. Eur J Cancer 36: 748-753.

55. Takahashi Y, Kitadai Y, Bucana CD, Cleary KR, Ellis LM (1995) Expression of vascular endothelial growth factor and its receptor, KDR, correlates with vascularity, metastasis, and proliferation of human colon cancer. Cancer Res 55: 3964-3968.

56. Kumar H, Heer K, Lee PW, Duthie GS, MacDonald AW, et al. (1998) Preoperative serum vascular endothelial growth factor can predict stage in colorectal cancer. Clin Cancer Res 4: 1279-1285.

57. Zhan P, Wang J, Lv XJ, Wang Q, Qu LX, et al. (2009) Prognostic value of vascular endothelial growth factor expression in patients with lung cancer: a systematic review with meta-analysis. J Thorac Oncol 4: 1094-1103.

58. Zhan P, Wang J, Lv XJ, Wang Q, Qu LX, et al. (1997) Expression of the angiogenic factors vascular endothelial cell growth factor, acidic and basic fibroblast growth factor, tumor growth factor beta-1, platelet-derived endothelial cell growth factor, placenta growth factor, and pleiotrophin in human primary breast cancer and its relation to angiogenesis. Cancer Res 57: 963-969

59. Olson TA, Mohanraj D, Carson LF, Ramakrishnan S (1994) Vascula permeability factor gene expression in normal and neoplastic human ovaries. Cancer Res 54: 276-280.

60. Plate KH, Breier G, Weich HA, Risau W (1992) Vascular endothelial growth factor is a potential tumour angiogenesis factor in human gliomas in vivo. Nature 359: 845-848.

61. Gerber HP, Ferrara N (2005) Pharmacology and pharmacodynamics of bevacizumab as monotherapy or in combination with cytotoxic therapy in preclinical studies. Cancer Res 65: 671-680.

62. Lenzer J (2011) FDA committee votes to withdraw bevacizumab for breast cancer. BMJ 343: d4244.

63. Heinrich MC, Maki RG, Corless CL, Antonescu CR, Harlow A, et al. (2008) Primary and secondary kinase genotypes correlate with the biological and clinical activity of sunitinib in imatinib-resistant gastrointestinal stromal tumor. $J$ Clin Oncol 26: 5352-5359.

64. Christensen JG (2007) A preclinical review of sunitinib, a multitargeted recepto tyrosine kinase inhibitor with anti-angiogenic and antitumour activities. Ann Oncol 18 Suppl 10: x3-10. 
Citation: French KJ, Frazier KS (2011) The Role of VEGF and VEGF Receptors in Carcinogenesis. J Carcinogene Mutagene S2:002. doi:10.4172/21572518.S2-002

65. Wilhelm S, Carter C, Lynch M, Lowinger T, Dumas J, et al. (2006) Discovery and development of sorafenib: a multikinase inhibitor for treating cancer. Nat Rev Drug Discov 5: 835-844.

66. GlaxoSmithKline Pharmaceuticals (2005) Votrient (pazopanib) NDA Pharmacology Review.

67. Genentech (2011) Avastin (bevacisumab) Prescribing Information.

68. Wu S, Chen JJ, Kudelka A, Lu J, Zhu X (2008) Incidence and risk of hypertension with sorafenib in patients with cancer: a systematic review and meta-analysis. Lancet Oncol 9: 117-123.

69. Bukowski RM (2010) Systemic therapy for metastatic renal cell carcinoma in treatment naive patients: a risk-based approach. Expert Opin Pharmacother 11: 2351-2362.

70. Yang R, Thomas GR, Bunting S, Ko A, Ferrara N, et al. (1996) Effects of vascular endothelial growth factor on hemodynamics and cardiac performance. J Cardiovasc Pharmacol 27: 838-844.

71. Ku DD, Zaleski JK, Liu S, Brock TA (1993) Vascular endothelial growth facto induces EDRF-dependent relaxation in coronary arteries. Am J Physiol 265: 586-592.

72. Curwen JO, Musgrove HL, Kendrew J, Richmond GH, Ogilvie DJ, et al (2008) Inhibition of vascular endothelial growth factor-a signaling induces hypertension: examining the effect of cediranib (recentin; AZD2171) treatment on blood pressure in rat and the use of concomitant antihypertensive therapy. Clin Cancer Res 14: 3124-3131.
73. Roodhart JM, Langenberg MH, Witteveen E, Voest EE (2008) The molecular basis of class side effects due to treatment with inhibitors of the VEGF/VEGFR pathway. Curr Clin Pharmacol 3: 132-143.

74. Sica DA (2006) Angiogenesis inhibitors and hypertension: an emerging issue $\mathrm{J}$ Clin Oncol 24: 1329-1331.

75. Hasinoff BB, Patel D (2010) The lack of target specificity of small molecule anticancer kinase inhibitors is correlated with their ability to damage myocytes in vitro. Toxicol Appl Pharmacol 249: 132-139.

76. Kumar R, Crouthamel MC, Rominger DH, Gontarek RR, Tummino PJ, et al (2009) Myelosuppression and kinase selectivity of multikinase angiogenesis inhibitors. Br J Cancer 101: 1717-1723.

77. Karaman MW, Herrgard S, Treiber DK, Gallant P, Atteridge CE, et al. (2008) A quantitative analysis of kinase inhibitor selectivity. Nat Biotechnol 26: 127-132.

78. Yamaguchi O, Watanabe T, Nishida K, Kashiwase K, Higuchi Y, et al. (2004) Cardiac-specific disruption of the c-raf-1 gene induces cardiac dysfunction and apoptosis. J Clin Invest 114: 937-943.

79. Force T, Krause DS, Van Etten RA (2007) Molecular mechanisms of cardiotoxicity of tyrosine kinase inhibition. Nat Rev Cancer 7: 332-344.

80. French KJ, Coatney RW, Renninger JP, Hu CX, Gales TL, et al. (2010) Differences in effects on myocardium and mitochondria by angiogenic inhibitors suggest separate mechanisms of cardiotoxicity. Toxicol Pathol 38: 691-702.
This article was originally published in a special issue, Growth factors : Carcinogenesis handled by Editor(s). Dr. Jaroslava Halper, The University of Georgia, USA; Dr. Ian Stuart Zagon, The Pennsylvania State University, USA 